Review paper

\title{
Hepatology topics of special interest from Central Europe (Czech Republic, Hungary, Poland, Slovakia)
}

\author{
Bela Hunyady', Jerzy Jaroszewicz², L'ubomír Lipták³, L'ubomír Skladaný3 , Jan Sperl ${ }^{4}$, Juraj Šváč \\ 'Somogy County Kaposi Mór Teaching Hospital, Kaposvár, Hungary \\ ${ }^{2}$ Department of Infectious Diseases and Hepatology, Medical University in Bialystok, Bialystok, Poland \\ ${ }^{3}$ HEGITO (Hepatology, Gastroenerology and Liver Transplant Division) of Department Internal Medicine II, Slovak Medical University BA, \\ F. D. Roosevelt University Hospital, Banska Bystrica, Slovakia \\ ${ }^{4}$ Department of Hepatogastroenterology, Institute for Clinical and Experimental Medicine, Prague, Czech Republic
}

\begin{abstract}
This compilation represents abstracts of presentations at the first meeting of the Initiative Group for the Central European Hepatologic Collaboration (CEHC, Warsaw, 19-21 November, 2015) on topics with special interest from four Central European countries and includes a summary of a boceprevir-based triple therapy cohort programme (from Hungary), current trends and challenges in liver diseases (from Poland), acute kidney injury in liver cirrhosis (from Slovakia) and treatment of hepatitis C in haemodialysed patients (from the Czech Republic). All these topics are or have been of high interest during the last several years, and many of them are still not completely resolved with the emergence and availability of the novel interferon-free therapies for hepatitis $C$ and with other advances in the pathophysiology and treatment of liver diseases.
\end{abstract}

Key words: boceprevir, direct acting antiviral drug, hepatitis C virus, interferon, kidney injury.

\section{Address for correspondence}

Bela Hunyady, Somogy County Kaposi Mór Teaching Hospital, Kaposvár, Hungary, e-mail: bhunyady@yahoo.com

\section{Introduction}

Abstracts on topics with special interest are reported in this paper as submitted by the experts from four countries to the first meeting of the initiative group of the Central European Hepatologic Collaboration (CEHC). The aim and scope of this collaboration were established at this initiative meeting in Warsaw, on 19-21 November, 2015, by invited experts from each country. Among other issues, results of a boceprevir-based triple therapy cohort programme (from Hungary), current trends and challenges in liver diseases (from Poland), acute kidney injury in liver cirrhosis (from Slovakia) and treatment of hepatitis C in haemodialysed patients (from the Czech Republic) were discussed and are presented here.

\section{Special topic 1: Boceprevir-based triple therapy in real world setting: lessons from the Hungarian Named Patient Programme}

Background: During 2011 and 2013, 155 Hungarian hepatitis $\mathrm{C}(\mathrm{HCV})$ genotype 1 infected patients with advanced liver fibrosis, who did not respond to prior peginterferon + ribavirin dual therapy, could start boceprevir-based triple therapy (best available in 20112013 in Hungary) in a Named Patient Programme.

Aims and methods: Efficacy and safety of the therapy were retrospectively assessed based on virological responses at 24 weeks after completion of therapy (sustained virological response - SVR). Frequency and type of therapy-related serious adverse events and of those leading to therapy discontinuation have also been analyzed.

Results: In an intent-to-treat analysis $39.4 \%$ patients (61/155) achieved an SVR. Sustained virological response rates were $59.5 \%, 41.4 \%$ and $22.9 \%$ SVR ( $p<0.05$ compared to the other two categories) among previous relapsers, partial responders and null-responders, respectively, and $52.5 \%$ vs. $31.3 \%(p<0.05$ compared to the non-cirrhotics) amongst non-cirrhotics versus cirrhotics, respectively. Six out of the 33 most difficult to cure patients (previous null responders and cirrhotics at the same time) have achieved an SVR (18.2\%). Frequency of early discontinuations due to insufficient 
virological responses was $31.1 \%$, and due to adverse events $10.3 \%$. Reported frequency of serious adverse events was $9.8 \%$. These events were anaemia, diarrhoea, depression, agranulocytosis, elevated aminotransferases, generalized dermatitis and severe gingivitis with loss of teeth, prolonged QT interval on ECG, generalized oedema and severe dyspnoea, uroinfection, exacerbation of Crohn's disease, Campylobacter pylori infection and unacceptable weakness with fatigue. Eight patients received transfusions, 4 patients erythropoietin and 1 granulocyte colony stimulating factor during therapy. No death has been reported.

Conclusions: With boceprevir-based triple therapy, a relevant proportion of hepatitis $\mathrm{C}$ infected patients with advanced liver fibrosis achieved a sustained viral response - some of whom would have potentially needed a liver transplant or been lost due to progression of liver disease without it. In this cohort, the side effects resembled those reported in registration studies, and resulted in therapy discontinuation with consequent treatment failure in a relevant number of patients. Efficacy and tolerability of boceprevir-based triple therapy are suboptimal, particularly for the most difficult to cure patient population.

\section{Special topic 2: Current trends and challenges in liver diseases in Poland}

Despite limited health expenditure in Poland, the mortality from liver disease and cirrhosis is comparable to other EU countries, with an age-standardized death rate per 100,000 population of 22 in males and 8 in females. Importantly, time trends in mortality have been rather stable in the last 30 years. Nevertheless, there are significant gaps in epidemiology and prevention of liver diseases in Poland. As for HBV infection, a similar number of new cases have been recorded each year (1500-2000 cases) for the last ten years. On the other hand, vaccination coverage of newborns in Poland is $>99 \%$ and only isolated cases of acute hepatitis B (HBV) in children are diagnosed. Taken together, these data and the fact that the majority (>90\%) of HBV infection cases are diagnosed at a chronic stage might suggest that there is a significant proportion of undiagnosed chronic hepatitis B cases with a need for larger screening studies and evaluation of risk factors [1]. This is illustrated by data on HBsAg prevalence in healthcare workers, which in 2013 was $1.2 \%$, almost twice as high as in first time blood donors [2]. Other important data were provided by a multicenter, ongoing study on distribution of HBV genotypes (EPIGENES, $n=243$ ), suggesting more diverse genotype distribution, especially geographically, then previously observed, with the novel-for-Poland genotype $\mathrm{H}$ of $\mathrm{HBV}$ being the third most common. Certainly, epidemiologic data for hepatitis B prevalence in Poland need to be updated.

Another significant factor for liver diseases morbidity and mortality in Poland is alcoholic liver disease (ALD). Alcohol consumption in Poland is above average for EU countries, with over 101 per year per capita consumed in 2012 according to World Health Organization (WHO) data [3]. Importantly, average alcohol consumption per capita has been increasing in the last 50 years in Poland. There are limited data on alcohol-associated liver mortality in Poland, although according to WHO data it may as high as $>14$ per 100,000 inhabitants, which is twice as high as HCVrelated liver mortality. Non-alcoholic fatty liver disease (NAFLD) and non-alcoholic steatohepatitis (NASH) are other significant factors for liver disease morbidity, with largely unknown prevalence in Poland. According to the WHO, the prevalence of obesity in Poland is over $23 \%$ in males and $26 \%$ in females, with metabolic syndrome affecting as many as $30-39 \%$ of the population [4]. It is a well-known risk factor for NAFLD. There are limited data on prevalence of NAFLD and NASH, but small single-centre studies suggest that F3-F4 fibrosis might affect over $20 \%$ of subjects with NASH [5]. Finally, all of the above-mentioned liver disorders are significant risk factors for hepatocellular carcinoma (HCC). The Polish National Cancer Registry reports a stable number of new HCC cases diagnosed in the last 10 years, between 700 and 900 per year. On the other hand, surprisingly mortality from HCC exceeds the number of new cases, suggesting poor adherence for HCC reporting but also late diagnosis of HCC at already advanced stages not allowing for successful therapy, including liver transplantation.

In summary, beside chronic hepatitis $C$, whose epidemiology is quite well known in Poland, there are significant gaps in data on prevalence and morbidity in liver disorders associated with alcohol and liver steatosis. Furthermore, although an effective national vaccination programme for $\mathrm{HBV}$ was introduced in Poland over 10 years ago, no significant decrease in overall HBV prevalence is noted, which might suggest a large number of undiagnosed cases. Further studies on the epidemiology and burden of liver diseases are urgently needed in Poland.

\section{Special topic 3: acute kidney injury (AKI) in liver cirrhosis}

Background: Since its introduction by the International Ascites Club (currently the International Club 
of Ascites - ICA) [6], type I hepatorenal syndrome (HRS, serum creatinine $\mathrm{CrS}>2.5 \mathrm{mg} / \mathrm{dl}$ ) and later acute renal failure (ARF, CrS increase of $>50 \%$ to $>1.5$ ) [7] have been the categories to classify, prognose, treat and study kidney function impairment in end-stage liver disease (ESLD) [8]. Limitations posed by this classification evolved into the need for earlier and more structured diagnostic criteria of kidney injury and led ESLD researchers to investigate the possibility to adopt the AKIN algorithm [9]; after many years and controversies, in December 2012 the ICA agreed on the set of proposed diagnostic criteria of AKI in ESLD with the aim to test them in different patient populations.

Aims, material and methods: To analyze the impact of the proposed AKI criteria on outcome of hospitalized patients with ESLD. Definitions: AKI $1=$ acute rise from baseline $(\Delta)$ in CrS of $>50 \%$; AKI 2 and $3=\Delta \mathrm{CrS}>100$ and $>200 \%$, respectively. Retrospective analysis of electronic records. Study period: January 1 to September 30, 2013. Inclusion criteria: adult, ESLD, hospitalized. Exclusion criteria: malignancy, lack of data. Complications: infection, bleeding, others.

Results: Final analysis was performed on data of 326 patients (pts) with median age 54 years (19-86); 123 female (38\%); aetiology of ESLD: 179 alcoholic LD (55\%), 66 NASH (11\%), 24 viral (7\%); medians: Child (CTPS) 9 points (5-14), MELD 16 (6-37), length of hospital stay 9.5 days $(0-45)$.

AKI criteria were fulfilled by 84 pts (26\%), with grade 1, 2 and 3 of 45 (54\%), 19 (23\%) and 20 (24\%) pts, respectively. Criteria of chronic kidney disease (CKD) were met by $26 \%$ of pts; AKI was diagnosed in more patients with underlying CKD $>3$ as compared to those without $\mathrm{CKD}$ or with $\mathrm{CKD} \leq 3$ ( 0.24 vs. 0.46 , $p<0.009)$. MELD of pts with AKI as compared to pts without AKI (20 vs. 14.5, $p<0.0004$ ). Incidence of complications: non-AKI vs. AKI-1, -2 and $-3=0.09$; $0.31 ; 0.63$ and 0.60 , respectively $(p<0.0001)$; mortality: $0.02 ; 0.11 ; 0.21$ and 0.55 , respectively $(p<0.0001)$.

Discussion: This retrospective study supports the notion that the diagnosis of AKI in patients with ESLD should follow the proposed criteria. Results compare well with those from similar studies [10] on AKI in ESLD with respect to incidence of AKI and its impact on outcome. At variance with data of Garcia-Tsao is much higher prevalence of CKD; furthermore, a relatively new finding deserving further study is increased risk of $\mathrm{AKI}$ in patients with $\mathrm{CKD}>3$.

The proposed criteria have been commented on recently and persist as a prominent focus of interest to the scientific community. The CEHC could address this challenge. AKI and its impact on outcome. At variance with data of Garcia-Tsao [10-15] is much higher prevalence of CKD; a relatively new finding deserving further study is increased risk of AKI in pts with CKD $>3$. The proposed criteria have been commented on recently and persist as a prominent focus of interest to the scientific community. The CEHC could address this challenge.

Conclusions: The proposed criteria are good predictors of morbidity and mortality in patients hospitalized with ESLD. The rates of complications and mortality increase with the degree of AKI.

\section{Special topic 4: HCV treatment in haemodialysed patients}

Dialysis has been the primary treatment for endstage renal disease (ESRD), but renal transplantation is now the preferred treatment approach as it confers a significant survival benefit over dialysis. HCV infection is more common in haemodialysis patients and consequently in renal transplant recipients than in the general population and represents an important cause of morbidity and mortality in kidney transplant recipients [16]. HCV-positive transplant recipients have lower survival rates after transplantation compared with HCV-negative recipients. In a meta-analysis, Fabrizi et al. demonstrated an independent, significant impact of HCV on mortality in transplant patients, with a summary relative risk estimate of 1.79 [17]. Morales et al. reported that mortality during the first 4 years after transplant was higher in $\mathrm{HCV}+$ versus HCVtransplant recipients: $6.1 \%$ vs. $4 \%$. HCV infection has also been associated with poorer renal allograft survival [18]. HCV infection is the leading cause of liver disease after kidney transplantation and is associated with an increase in mortality. Chronic hepatitis and its sequelae are the main forms of liver disease in these patients. In addition, fibrosing cholestatic hepatitis, characterized by severe cholestasis with rapidly progressive liver failure, has been reported. Treatment of $\mathrm{HCV}$ in renal transplant recipients using standard interferon-based regimens was associated with high rates of renal allograft failure. Therefore, IFN-based therapy for $\mathrm{HCV}$ in kidney transplant recipients was recommended only in fibrosing cholestatic hepatitis with high risk of liver failure.

Goodkin et al. demonstrated that interferon-based treatment of kidney transplant candidates during the period of maintenance haemodialysis never became a standard approach in lowering the risk associated with HCV infection after kidney transplantation [19]. The study included almost fifty thousand patients on maintenance haemodialysis, and the overall prevalence of HCV positivity was $9.5 \%$. The prevalence ranged 
between $3.3 \%$ and $16.8 \%$. The study documented that the proportion of $\mathrm{HCV}$ patients referred for antiviral treatment (1\%) and kidney transplantation (3.7\%) was unsatisfactory. The main reason for treatment deferral was low efficacy and poor tolerability of interferonbased regimens. The SVR rate ranged between 30\% and $90 \%$ in genotype 1 patients and was closely dependent on the selection criteria.

Interferon-free DAA regimens provide higher efficacy and tolerability and are an attractive therapeutic option for haemodialysed patients. However, clinical data on the efficacy and safety of these new regimens are still limited. Sofosbuvir is renally cleared and its metabolites increased 13.8-fold to 21.7-fold in haemodialysed patients, depending on whether the drug was dosed before or after haemodialysis [20]. Optimal dosing of sofosbuvir in haemodialysed subjects is unknown and sofosbuvir is not approved for these patients. However, use of a reduced dose of sofosbuvir with daclatasvir or simeprevir has recently been reported in small cohorts. Combination of ombitasvir/ paritaprevir/ritonavir plus dasabuvir is an option for genotype 1 patients since all drugs are cleared by the liver. The RUBY- 1 study evaluated this combination in 20 patients with ESRD, most of them on haemodialysis [21]. The combination was well tolerated, and the overall SVR12 rate was 95\%. Combination of grazoprevir/elbasvir has been evaluated in the C-SURFER study, which included 116 genotype 1, CKD stage $4 / 5$ patients, $76 \%$ of them dialysis-dependent [22]. The combination was well tolerated, with an SVR12 rate of $99 \%$. There are no data on efficacy in other HCV genotypes so far.

\section{Discussion}

These selected topics by four experts from Central European countries point to many unresolved issues in the field of liver diseases as well as to similarities among these countries.

Based on the Hungarian experience of a boceprevir-based triple therapy cohort programme, only a proportion of hepatitis $\mathrm{C}$ infected patients with advanced liver fibrosis and previous treatment failure with dual therapy could achieve a sustained viral response (39.4\%). Both efficacy and tolerability of the boceprevir-based triple therapy have been found suboptimal, particularly for the most difficult to cure patients.

In Poland - beside chronic hepatitis $\mathrm{C}$, the epidemiology of which is quite well known - there are significant gaps in data on prevalence and morbidity of liver disorders associated with alcohol and liver steatosis. Furthermore, although an effective national vacci- nation programme for $\mathrm{HBV}$ was introduced more than 10 years ago, no significant decrease in overall $\mathrm{HBV}$ prevalence has been noted.

The retrospective study from Slovakia supports the notion that the diagnosis of AKI in patients with ESLD should follow the criteria proposed by the International Ascites Club. Results from Poland compare well with those from other similar studies on AKI in ESLD. The CEHC could address these challenges in the definition and predictive factors of AKI and could potentially evaluate its impact on outcome in a regional programme.

Based on the data explored by Prof. Sperl from the Czech Republic, HCV infection is more common in haemodialysis patients and consequently in renal transplant recipients than in the general population, and it represents an important cause of morbidity and mortality in kidney transplant recipients. Recent advances in the treatment of HCV may help to improve quality of life and life expectancy of haemodialysed patients with HCV infection. However, clinical data on the efficacy and safety of new regimens are still limited.

All these topics are, have been, or potentially will be of high interest in the Central European region, and offer grounds for joint, collaborative research and clinical studies for the countries concerned.

\section{Disclosure}

Authors report no conflict of interest.

\section{References}

1. Stępień M, Czarkowski MP. Hepatitis B in Poland in 2011. Przegl Epidemiol 2013; 67: 239-245.

2. Rybacki M, Piekarska A, Wiszniewska M, et al. Hepatitis B and C infection: is it a problem in Polish healthcare workers? Int J Occup Med Environ Health 2013; 26: 430-439.

3. World Health Organization. Global Status Report on Alcohol and Health, 2014. http://apps.who.int/iris/bitstream/10665/112736/ 1/9789240692763_eng.pdf?ua $=1$

4. Janszky I, Vatten L, Romundstad L, et al. Metabolic syndrome in Poland - POND study. Ann Agric Environ Med 2011; 18: 270272.

5. Raszeja-Wyszomirska J, Kurzawski G, Lawniczak M, et al. Nonalcoholic liver disease and HFE mutation: a Polish study. World J Gastroenterol 2010; 16: 2531-2536.

6. Arroyo V, Ginès P, Gerbes AL, et al. Definition and diagnostic criteria of refractory ascites and hepatorenal syndrome in cirrhosis. International Ascites Club. Hepatology 1996; 23: 164-176.

7. Salerno F, Gerbes A, Ginès P, et al. Diagnosis, prevention and treatment of hepatorenal syndrome in cirrhosis. Gut 2007; 56: 1310-1318.

8. European Association for the Study of the Liver. EASL clinical practice guidelines on the management of ascites, spontaneous bacterial peritonitis, and hepatorenal syndrome in cirrhosis. J Hepatol 2010; 53: 397-417. 
9. Wong F, Nadim MK, Kellum JA, et al. Working Party proposal for a revised classification system of renal dysfunction in patients with cirrhosis. Gut 2011; 60: 702-709.

10. Garcia-Tsao G, Parikh CR, Viola A. Acute kidney injury in cirrhosis. Hepatology 2008; 48: 2064-2077.

11. Belcher JM, Garcia-Tsao G, Sanyal AJ, et al. Association of AKI with mortality and complications in hospitalized patients with cirrhosis. Hepatology 2013; 57: 753-762.

12. Fagundes C, Barreto R, Guevara M, et al. A modified acute kidney injury classification for diagnosis and risk stratification of impairment of kidney function in cirrhosis. J Hepatol 2013; 59: 474-481.

13. Piano S, Rosi S, Maresio G, et al. Evaluation of the Acute Kidney Injury Network criteria in hospitalized patients with cirrhosis and ascites. J Hepatol 2013; 59: 482-489.

14. Cholongitas E, Calvaruso V, Senzolo M, et al. RIFLE classification as predictive factor of mortality in patients with cirrhosis admitted to intensive care unit. J Gastroenterol Hepatol 2009; 24: 1639-1647.

15. Altamirano J, Fagundes C, Dominguez M, et al. Acute kidney injury is an early predictor of mortality for patients with alcoholic hepatitis. Clin Gastroenterol Hepatol 2012; 10: 65-71.e3.

16. Sperl J, Frankova S, Senkerikova R, et al. Relevance of low viral load in haemodialysed patients with chronic hepatitis $\mathrm{C}$ virus infection. World J Gastroenterol 2015; 21: 5496-5504.

17. Fabrizi F, Martin P, Dixit V, Messa P. Hepatitis C virus infection and kidney disease: a meta-analysis. Clin J Am Soc Nephrol 2012; 7: 549-557.

18. Morales JM, Marcén R, Andres A, et al. Renal transplantation in patients with hepatitis $\mathrm{C}$ virus antibody. A long national experience. NDT Plus 2010; 3 (Suppl 2): ii41-ii46.

19. Goodkin DA, Bieber B, Gillespie B, et al. Hepatitis C infection is very rarely treated among hemodialysis patients. Am J Nephrol 2013; 38: 405-412.

20. Nazario HE, Ndungu M, Modi AA. Sofosbuvir and simeprevir in hepatitis $C$ genotype 1 - patients with end-stage renal disease on hemodialysis or GFR $>30 \mathrm{ml} / \mathrm{min}$. Liver Int 2015 Nov 19 . doi: 10.1111/liv.13025 [Epub ahead of print].

21. Pockros PJ, Reddy KR, Mantry PS, et al. Safety of ombitasvir/ paritaprevir/ritonavir plus dasabuvir for treating HCV GT1 infection in patients with severe renal impairment or end-stage renal-disease: The Ruby-I Study. AASLD, San Francisco 2015.

22. Roth D, Nelson DR, Bruchfeld A, et al. Grazoprevir plus elbasvir in treatment-naive and treatment-experienced patients with hepatitis $C$ virus genotype 1 infection and stage 4-5 chronic kidney disease (the C-SURFER study): a combination phase 3 study. Lancet 2015; 386: 1537-1545. 